\title{
EDITORIAL INFORMATION 2017
}

The 2017 Nominata reflects the intense work developed during the year, composing the period from January to October. The number of submissions remained almost the same. Once again, A\&FR was the highlight in the SPELL ranking for 2015, where we are the top-ranked Journal in the area of Administration and Accounting, when considered impact for two years, and stand at the second place, when considered five years. A qualitative change resulted from a lot of joint effort was the anticipation of the editions' release date to the beginning of every four months, instead of the end of this period. The plans for 2018 involve expanding the theoretical essay as an alternative of additional communication to the theoretical-empirical work, greater availability of blog posts and effort to reduce publication deadlines by lines of research.

I would like to thank the authors, reviewers, readers, Associate Editors, Ad-Hoc Editors, Editorial Board members, service providers and editorial assistants who had the work developed with professionalism, confidentiality, respect, and great affection for the generation of knowledge in the scope of A\&FR.

The Ad-Hoc Editors correspond to a recent participation experience of researchers who acted as editors specifically for some articles submitted by professors near to the Scientific Committee or for cases of great specificity.

In 2017, we add the role of Ad-Hoc Editors by lines of research: Claudio Wanderley, Marcia Mendes De Luca, Bruno Salotti, Andrea Maria Accioly Fonseca Minardi, Raquel de Freitas Oliveira, and Francisco Henrique Figueiredo de Castro Junior. Also, we had the contribution of Ad-Hoc Editor by article. In this particular I would like to thank to Joséte Florêncio dos Santos e Marcelo Francini Girão Barroso.

We have financial support from FIPECAFI, SIBi-USP, CNPq, and CAPES, which provided the activities development within the established challenges. Similarly, I would like to thank our service providers who contributed to the accomplishment of our work with professionalism and attention. We refer specifically to Eduarda Bodaneze de Oliveira, Eliane Simões Pereira, Evandro Lisboa Freire, Luiza Carvalho, José Couto, Kris Gillespie, Letra1 Serviços Editoriais, Libre Traduções, and SOStexto.

I am particularly grateful to SciELO for its support in improving the ScholarOne management control system, which provided a significant change in the management platform for the Journal. In this sense, the support given to the implementation and improvement of the system was vital. Back in the year of 2015, we were accepted by Scopus and this new indexation seems to be very promising in terms of external visibility. We received 152 articles submitted between January and October, 2017, with 127 reviewers analyzing 68 researches, which provided a desk rejection of $55 \%$ and a total rejection of $62 \%$. We had 60 new reviewers incorporated into the group that provided demand response. We published 25 original articles and 1 original essay in editions 73,74 , and 75 . It is very relevant for A\&FR to participate in the different lines of research and the distribution is emphasized in the lines of Accounting for External Users and Markets.

Fábio Frezatti

Editor-in-Chief 
Table 1 Lines of research

\begin{tabular}{|c|c|c|c|c|}
\hline Line of Research & Submission & Percentage & Publication & Percentage \\
\hline $\begin{array}{c}\text { Controllership and } \\
\text { Management Accounting }\end{array}$ & 30 & $19.7 \%$ & 1 & $4.0 \%$ \\
\hline $\begin{array}{l}\text { Accounting for } \\
\text { External Users }\end{array}$ & 69 & $45.4 \%$ & 13 & $50.0 \%$ \\
\hline $\begin{array}{l}\text { Markets: Financial, } \\
\text { Credit, and Capital }\end{array}$ & 39 & $25.7 \%$ & 8 & $30.0 \%$ \\
\hline $\begin{array}{c}\text { Accounting, Finance } \\
\text { and Actuarial Education } \\
\text { and Research }\end{array}$ & 10 & $6.6 \%$ & 2 & $8.0 \%$ \\
\hline Actuarial Sciences & 4 & $2.6 \%$ & 2 & $8.0 \%$ \\
\hline $\begin{array}{l}\text { Emerging Themes in } \\
\text { Accounting, Finance and } \\
\text { Actuarial Sciences }\end{array}$ & 0 & $0.0 \%$ & 0 & $0.0 \%$ \\
\hline Total & 152 & $100.0 \%$ & 26 & $100.0 \%$ \\
\hline
\end{tabular}

Table 2 Authors' geographic range

\begin{tabular}{ccccc}
\hline \multirow{2}{*}{ Authors' affiliation } & \multicolumn{2}{c}{ Submission } & \multicolumn{2}{c}{ Publication } \\
\cline { 2 - 5 } & Frequency & Percentage & Frequency & Percentage \\
\hline FEA-USP (São Paulo) & 16 & $3.8 \%$ & 17 & $9.2 \%$ \\
\hline São Paulo (but FEA-USP) & 72 & $17.1 \%$ & 40 & $25.6 \%$ \\
\hline Other Brazilian States & 270 & $64.0 \%$ & 3 & $60.6 \%$ \\
\hline International & 64 & $15.1 \%$ & $\mathbf{6 3}$ & $4.6 \%$ \\
\hline Total & $\mathbf{4 2 2}$ & $\mathbf{1 0 0 . 0} \%$ & $\mathbf{1 0 0 . 0} \%$ \\
\hline
\end{tabular}

Table 3 Publication demand

\begin{tabular}{ccc}
\hline & & Jan./Oct. 2017 \\
\cline { 2 - 3 } & Frequency & Percentage \\
\hline Received & 49 & $24.4 \%$ \\
Total & 152 & $75.6 \%$ \\
Approved & $\mathbf{2 0 1}$ & $\mathbf{1 0 0 . 0} \%$ \\
Reproved during review & 27 & $13.0 \%$ \\
Rejected before review (desk) & 24 & $11.5 \%$ \\
Withdrawal at the request of the authors & 84 & $40.4 \%$ \\
Total of editorial decisions & 7 & $3.4 \%$ \\
Awaiting editor's review & $\mathbf{1 4 2}$ & $\mathbf{7 0 . 5 \%}$ \\
Awaiting reviewer's scores & 20 & $9.6 \%$ \\
Awaiting authors' revision & 29 & $13.9 \%$ \\
Final outcome of articles in $\mathbf{2 0 1 7}$ & 10 & $4.8 \%$ \\
\hline
\end{tabular}




\section{Schedule of Reviewers}

Adrian Heringer Pizzinga

UFF - Universidade Federal Fluminense

Adriana Maria Araújo

USP - Universidade de São Paulo - Campus Ribeirão Preto

Adriano Leal Bruni

UFBA - Universidade Federal da Bahia

Alessandra Carvalho de Vasconcelos

UFC - Universidade Federal do Ceará

Alexandre de Pádua Carrieri

UFMG - Universidade Federal de Minas Gerais

Alexandre Sanches Garcia

FECAP - Fundação Escola de Comércio Álvares Penteado

Amaury José Rezende

USP - Universidade de São Paulo - Campus Ribeirão Preto

Ana Carolina Maia

USP - Universidade de São Paulo - Campus Capital

Anderson Luiz Rezende Mol

UFRN - Universidade Federal do Rio Grande do Norte

André Carlos Busanelli de Aquino

USP - Universidade de São Paulo - Campus Ribeirão Preto

André Luiz Bufoni

UFRJ - Universidade Federal do Rio de Janeiro

Andrea Maria Accioly Fonseca Minardi

INSPER - Instituto de Ensino e Pesquisa

Andson Braga de Aguiar

USP - Universidade de São Paulo - Campus Capital

Antonio Carlos Dias Coelho

UFC - Universidade Federal do Ceará

Antônio Gualberto Pereira

UFBA - Universidade Federal da Bahia

Antonio Marcos Duarte Júnior

UERJ - Universidade Estadual do Rio de Janeiro 
Ariovaldo dos Santos

USP - Universidade de São Paulo - Campus Capital

Arlindo Carvalho Rocha

UDESC - Universidade do Estado de Santa Catarina

Aureliano Angel Bressan

UFMG - Universidade Federal de Minas Gerais

Benjamin Miranda Tabak

UCB - Universidade Católica de Brasília

Bernardo Lanza Queiroz

UFMG - Universidade Federal de Minas Gerais

Bruno Dore Rodrigues

UFJF - Universidade Federal de Juiz de Fora

Bruno Ferreira Frascaroli

UFPB - Universidade Federal da Paraíba

Bruno José Machado de Almeida

ISCAC - Instituto Superior de Contabilidade e Administração de Coimbra

Bruno Meirelles Salotti

USP - Universidade de São Paulo - Campus Capital

Bruno Paula Rocha

UFABC - Universidade Federal do ABC

Carlos Alberto Diehl

UNISINOS - Universidade do Vale do Rio dos Sinos

Carlos Eduardo Pimentel

UnB - Universidade de Brasília

Carlos Heitor Campani

UFRJ - Universidade Federal do Rio de Janeiro

Carlos Henrique Silva do Carmo

UFG - Universidade Federal de Goiás

Carlos Denner Santos Junior

UnB - Universidade de Brasília

César Medeiros Cupertino

FUCAPE - Fundação Instituto Capixaba de Pesquisas em Contabilidade, Economia e Finanças

Claudia Ferreira da Cruz

UFRJ - Universidade Federal do Rio de Janeiro 
Claudio Henrique da Silveira Barbedo

IBMEC - Instituto Brasileiro de Mercado de Capitais

Cristiane Silva Corrêa

UFRN - Universidade Federal do Rio Grande do Norte

Daniel Magalhães Mucci

USP - Universidade de São Paulo - Campus Capital

Daniel Reed Bergmann

USP - Universidade de São Paulo - Campus Capital

\section{Dany Rogers Silva}

UFU - Universidade Federal de Uberlândia

Denis Forte

UPM - Universidade Presbiteriana Mackenzie

\section{Diana Vaz de Lima}

UnB - Universidade de Brasília

Edgard Bruno Cornacchione Júnior

USP - Universidade de São Paulo - Campus Capital

\section{Edilson Paulo}

UFPR - Universidade Federal do Paraná

\section{Eduardo da Silva Flores}

USP - Universidade de São Paulo - Campus Capital

Eduardo Pozzi Lucchesi

FECAP - Fundação Escola de Comércio Álvares Penteado

Edvalda Araújo Leal

UFU - Universidade Federal de Uberlândia

\section{Elionor Farah Jreige Weffort}

FECAP - Fundação Escola de Comércio Álvares Penteado

\section{Eliseu Martins}

USP - Universidade de São Paulo - Campus Ribeirão Preto

\section{Elmo Tambosi Filho}

UMESP - Universidade Metodista de São Paulo

\section{Fabiano Guasti Lima}

USP - Universidade de São Paulo - Campus Ribeirão Preto

\section{Fabiano Maury Raupp}

UDESC - Universidade do Estado de Santa Catarina 
Fabio Garcia Gallo

FGV - Fundação Getúlio Vargas - Campus São Paulo

Fernando Caio Galdi

FUCAPE Business School - ES

Fernando Chiqueto da Silva

IASB - International Accounting Standards Board

Fernando de Holanda Barbosa Filho

FGV - Fundação Getúlio Vargas - Campus Rio de Janeiro

Flávia de Souza Ávila

ESPM - Escola Superior de Propaganda e Marketing

Flávio Henrique Miranda de Araújo Freire

UFRN - Universidade Federal do Rio Grande do Norte

Gabriel Filipe Rodrigues Vasconcelos

PUC - Pontifícia Universidade Católica do Rio de Janeiro

Gilberto José Miranda

UFU - Universidade Federal de Uberlândia

\section{Guilherme Kirch}

UFRGS - Universidade Federal do Rio Grande do Sul

Guillermo Oscar Braumbeck

USP - Universidade de São Paulo - Campus Capital

Faculdade Fipecafi

FGV - Fundação Getúlio Vargas - Campus São Paulo

\section{Heloisa Helena Nascimento Oliveira}

Tribunal de Contas do Estado de Minas Gerais

\section{Herbert Kimura}

UnB - Universidade de Brasília

Ilse Maria Beuren

UFPR - Universidade Federal do Paraná

Isabel Maria Estima Costa Lourenço

IUL - Instituto Universitário de Lisboa

João Vinícius Carvalho

USP - Universidade de São Paulo - Campus Capital

UNIFESP - Universidade Federal de São Paulo

José Alonso Borba

UFSC - Universidade Federal de Santa Catarina 
José Carlos Tiomatsu Oyadomari

UPM - Universidade Presbiteriana Mackenzie

INSPER - Instituto de Ensino e Pesquisa

José Elias Feres de Almeida

UFES - Universidade Federal do Espírito Santo

José Roberto Ferreira Savóia

USP - Universidade de São Paulo - Campus Capital

Laura Taboada Pinheiro

UFMG - Universidade Federal de Minas Gerais

Lucas Ayres Barreira de Campos Barros

USP - Universidade de São Paulo - Campus Capital

Lúcia Lima Rodrigues

UM - Universidade do Minho

Luiz Antonio Abrantes

UFV - Universidade Federal de Viçosa

Luiz Alberton

UFSC - Universidade Federal de Santa Catarina

Luiz Felipe de Araújo Pontes Girão

UFPB - Universidade Federal da Paraíba

Luiz Carlos Jacob Perera

UPM - Universidade Presbiteriana Mackenzie

Maisa de Souza Ribeiro

USP - Universidade de São Paulo - Campus Ribeirão Preto

Mamadou Dieng

UEPB - Universidade Estadual da Paraíba

Marcelo Álvaro da Silva Macedo

UFRJ - Universidade Federal do Rio de Janeiro

\section{Marcelo Cabus Klotzle}

PUC - Pontifícia Universidade Católica do Rio de Janeiro

\section{Marcelo Francini Girão Barroso}

UPM - Universidade Presbiteriana Mackenzie

Marcelo José Braga

UFV - Universidade Federal de Viçosa

Márcia Martins Mendes De Luca

UFC - Universidade Federal do Ceará 
Márcio André Veras Machado

UFPB - Universidade Federal da Paraíba

Marcos Soares da Silva

BCB - Banco Central do Brasil

Maria João Major

NOVA - Universidade Nova de Lisboa

Mario António Gomes Augusto

UC - Universidade de Coimbra

Mateus Alexandre Costa dos Santos

UFPB - Universidade Federal da Paraíba

Nálbia de Araújo Santos

UFV - Universidade Federal de Viçosa

Natalie Haanwinckel Hurtado

SUSEP - Superintendência de Seguros Privados

Orleans Silva Martins

UFPB - Universidade Federal da Paraíba

Otávio Ribeiro de Medeiros

UnB - Universidade de Brasília

Pablo Rogers Silva

UFU - Universidade Federal de Uberlândia

Patrícia de Souza Costa

UFU - Universidade Federal de Uberlândia

Patrícia Siqueira Varela

USP - Universidade de São Paulo - Campus Capital

Paulo Augusto Pettenuzzo de Britto

UnB - Universidade de Brasília

Paulo Roberto Barbosa Lustosa

UnB - Universidade de Brasília

Paulo Roberto da Cunha

FURB - Fundação Universidade Regional de Blumenau

Rafael Barreiros Porto

UnB - Universidade de Brasília

Rafael Felipe Schiozer

FGV - Fundação Getúlio Vargas - Campus Capital 
Rafael Morais de Souza

UFMG - Universidade Federal de Minas Gerais

Reinaldo Guerreiro

USP - Universidade de São Paulo - Campus Capital

Renata Turola Takamatsu

UFMG - Universidade Federal de Minas Gerais

\section{Ricardo José de Almeida}

INSPER - Instituto de Ensino e Pesquisa

\section{Ricardo Ratner Rochman}

FGV - Fundação Getúlio Vargas - Campus Capital

Ricardo Rocha Azevedo

UFU - Universidade Federal de Uberlândia

\section{Robert Iquiapaza}

UFMG - Universidade Federal de Minas Gerais

\section{Roberto Borges Kerr}

UPM - Universidade Presbiteriana Mackenzie

\section{Roberto Carlos Klann}

FURB - Universidade Regional de Blumenau

Rodrigo Fernandes Malaquias

UFU - Universidade Federal de Uberlândia

Rogério Nagamine Costanzi

MPS - Ministério da Previdência Social

\section{Romualdo Douglas Colauto}

UFPR - Universidade Federal do Paraná

\section{Rosana Tavares}

USP - Universidade de São Paulo - Campus Capital

\section{Rosimeire Pimentel Gonzaga}

UFMG - Universidade Federal de Minas Gerais

\section{Rui Domingos Ribeiro da Cunha Marques}

ULisboa - Universidade de Lisboa

\section{Sady Mazzioni}

Unochapecó - Universidade Comunitária da Região de Chapecó

\section{Sérgio Leão}

BCB - Banco Central do Brasil 


\section{Sheizi Calheira de Freitas}

UFBA - Universidade Federal da Bahia

Silvia Pereira de Castro Casa Nova

USP - Universidade de São Paulo - Campus Capital

\section{Silvia Russi de Domenico}

UPM - Universidade Presbiteriana Mackenzie

\section{Silvio Hiroshi Nakao}

USP - Universidade de São Paulo - Campus Ribeirão Preto

\section{Sonia Valle Walter Borges de Oliveira}

USP - Universidade de São Paulo - Campus Ribeirão Preto

Tatiana Albanez

USP - Universidade de São Paulo - Campus Capital

Vinicio de Souza e Almeida

UFRN - Universidade Federal do Rio Grande do Norte

\section{Wagner Moura Lamounier}

UFMG - Universidade Federal de Minas Gerais

\section{Wilson Toshiro Nakamura}

UPM - Universidade Presbiteriana Mackenzie

Wlademir Ribeiro Prates

UFSC - Universidade Federal de Santa Catarina

\section{Index by Title}

Accounting History Research: Scope, Topics and Agenda

CARMONA, Salvador

v.28, n.75, p.321-325, set./dez.2017, DOI: 10.1590/1808-057x201790210

Accounting research: between natural science and practice

HARTMANN, Frank G. H.

v.28, n.73, p.7-10, Jan./Apr.2017, DOI: 10.1590/1808-057x201790160

Challenges with the public policy of measuring assets to set tariffs in the electricity sector: should someone benefit and someone be sacrificed?

ANDRADE, Maria Elisabeth Moreira Carvalho; MARTINS, Eliseu

v.28, n.75, p.344-360, Sep./Dec.2017, DOI: 10.1590/1808-057x201703160

Cognitive brain mapping of auditors and accountants in going concern judgments

CARVALHO JÚNIOR, César Valentim de Oliveira; CORNACCHIONE, Edgard; ROCHA, Armando Freitas da; ROCHA, Fábio Theoto

v.28, n.73, p.132-147, Jan./Apr.2017, DOI: 10.1590/1808-057x201703430 
Complexity and corporate governance: an analysis of companies listed on the BM\&FBOVESPA

ASSUNÇÃO, Renata Rouquayrol; LUCA, Márcia Martins Mendes De; VASCONCELOS, Alessandra Carvalho de v.28, n.74, p.213-228, May/Aug.2017, DOI: 10.1590/1808-057x201702660

The effect of asymmetric information risk on returns of stocks traded on the BM\&FBOVESPA

SIQUEIRA, Leonardo Souza; AMARAL, Hudson Fernandes; CORREIA, Laíse Ferraz

v.28, n.75, p.425-444, Sep./Dec.2017, DOI: 10.1590/1808-057x201705230

Effects of IFRS adoption on tax avoidance

BRAGA, Renata Nogueira

v.28, n.75, p.407-424, Sep./Dec.2017, DOI: 10.1590/1808-057x201704680

Effects of the audit committee and the fiscal council on earnings quality in Brazil

BAIOCO, Vitor Gomes; ALMEIDA, José Elias Feres de

v.28, n.74, p.229-248, May/Aug.2017, DOI: 10.1590/1808-057x201703250

Executive branch federal civil servant mortality by sex and educational level - 1993/2014

BELTRÃO, Kaizo Iwakami; SUGAHARA, Sonoe

v.28, n.75, p.445-464, Sep./Dec.2017, DOI: 10.1590/1808-057x201704320

Exposition to Factors of the Investment Funds Market in Brazil

MAESTRI, Cláudia Olímpia Neves Mamede; MALAQUIAS, Rodrigo Fernandes

v.28, n.73, p.61-76, Jan./Apr.2017, DOI: 10.1590/1808-057x201702940

Geographically Weighted Logistic Regression Applied to Credit Scoring Models

ALBUQUERQUE, Pedro Henrique Melo; MEDINA, Fabio Augusto Scalet; SILVA, Alan Ricardo da v.28, n.73, p.93-112, Jan./Apr.2017, DOI: 10.1590/1808-057x201703760

Heterogeneity in earnings quality between different classes of companies after IFRS adoption: evidence from Brazil BLACK, Roberto; NAKAO, Sílvio Hiroshi v.28, n.73, p.113-131, Jan./Apr.2017, DOI: 10.1590/1808-057x201702750

ICPC 14: what is missing?

SANTOS, Ariovaldo dos; LONDERO, Paola R.

v.28, n.75, p.478-485, Sep./Dec.2017, DOI: 10.1590/1808-057x201704740

IFRS, synchronicity, and financial crisis: the dynamics of accounting information for the Brazilian capital market FIGLIOLI, Bruno; LEMES, Sirlei; LIMA, Fabiano Guasti

v.28, n.75, p.326-343, Sep./Dec.2017, DOI: 10.1590/1808-057x201704450

Impacts of the regulatory model for market risk capital: application in a special savings company, an insurance company, and a pension fund

CHAN, Betty Lilian; MARQUES, Felipe Tumenas

v.28, n.75, p.465-477, Sep./Dec.2017, DOI: 10.1590/1808-057x201703840

Influence of board interlocking on earnings management

CUNHA, Paulo Roberto da; PICCOLI, Marcio Roberto

v.28, n.74, p.179-196, May/Aug.2017, DOI: 10.1590/1808-057x201701980 
Investor relations and information asymmetry

RODRIGUES, Sandrielem da Silva; GALDI, Fernando Caio

v.28, n.74, p.297-312, May/Aug.2017, DOI: 10.1590/1808-057x201703630

Positivism and "alternative" accounting research

MAJOR, Maria João

v.28, n.74, p.173-178, maio/ago.2017, DOI: 10.1590/1808-057x201790190

Predicting financial distress in publicly-traded companies

REZENDE, Felipe Fontaine; MONTEZANO, Roberto Marcos da Silva; OLIVEIRA, Fernando Nascimento de; LAMEIRA, Valdir de Jesus

v.28, n.75, p.390-406, Sep./Dec.2017, DOI: 10.1590/1808-057x201704460

Predictive power of Brazilian equity fund performance using $\mathrm{R}^{2}$ as a measure of selectivity

GUZELLA, Marcelo dos Santos; CAMPANI, Carlos Heitor

v.28, n.74, p.282-296, May/Aug.2017, DOI: 10.1590/1808-057x201703590

The relationship between the performance and legal form of microfinance institutions

COSTA, Ruan Rodrigo Araújo da

v.28, n.75, p.377-389, Sep./Dec.2017, DOI: 10.1590/1808-057x201703660

Satisfaction among accounting professors in Brazil

ARAÚJO, Tamires Sousa; MIRANDA, Gilberto José; PEREIRA, Janser Moura

v.28, n.74, p.264-281, May/Aug.2017, DOI: 10.1590/1808-057x201703420

Social quotas, affirmative actions, and dropout in the Business field: empirical analysis in a Brazilian federal university CAMPOS, Larissa Couto; MACHADO, Thalyson Renan Bitencourt; MIRANDA, Gilberto José; COSTA, Patrícia de Souza

v.28, n.73, p.27-42, Jan./Apr.2017, DOI: 10.1590/1808-057x201702590

Unconditional conservatism in Brazilian public companies and tax neutrality

VALE, Juliana Pinhata Sanches do; NAKAO, Sílvio Hiroshi

v.28, n.74, p.197-212, May/Aug.2017, DOI: 10.1590/1808-057x201702450

Using the Newcomb-Benford Law as a Deviation Identification Method in Continuous Auditing Environments: A Proposal for Detecting Deviations over Time

SILVA, Wilton Bernardino da; TRAVASSOS, Silvana Karina de Melo; COSTA, Jose Isidio de Freitas

v.28, n.73, p.11-26, Jan./Apr.2017, DOI: 10.1590/1808-057x201702690

Value-at-risk modeling and forecasting with range-based volatility models: empirical evidence

MACIEL, Leandro dos Santos; BALLINI, Rosangela

v.28, n.75, p.361-376, Sep./Dec.2017, DOI: 10.1590/1808-057x201704140

Value Relevance vis-à-vis Disclosure on Business Combinations and Goodwill Recognized by Publicly Traded Brazilian Companies

SOUZA, Maíra Melo de; BORBA, José Alonso

v.28, n.73, p.77-92, Jan./Apr.2017, DOI: 10.1590/1808-057x201703240

Voluntary disclosure and earnings management: evidence from the Brazilian capital market

CONSONI, Silvia; COLAUTO, Romualdo Douglas; LIMA, Gerlando Augusto Sampaio Franco de

v.28, n.74, p.249-263, May/Aug.2017, DOI: 10.1590/1808-057x201703360 
Why has not it worked? An empirical application of the extended Burns and Scapens' framework within the implementation of a controlling department

ESPEJO, Márcia Maria dos Santos Bortolocci; EGGERT, Neusa Sawczuk von

v.28, n.73, p.43-60, Jan./Apr.2017, DOI: 10.1590/1808-057x201702820

\section{Index by Author}

ALBUQUERQUE, Pedro Henrique Melo

Geographically Weighted Logistic Regression Applied to Credit Scoring Models

v.28, n.73, p.93-112, Jan./Apr.2017, DOI: 10.1590/1808-057x201703760

ALMEIDA, José Elias Feres de

Effects of the audit committee and the fiscal council on earnings quality in Brazil

v.28, n.74, p.229-248, May/Aug.2017, DOI: 10.1590/1808-057x201703250

AMARAL, Hudson Fernandes

The effect of asymmetric information risk on returns of stocks traded on the BM\&FBOVESPA

v.28, n.75, p.425-444, Sep./Dec.2017, DOI: 10.1590/1808-057x201705230

ANDRADE, Maria Elisabeth Moreira Carvalho

Challenges with the public policy of measuring assets to set tariffs in the electricity sector: should someone benefit and someone be sacrificed?

v.28, n.75, p.344-360, Sep./Dec.2017, DOI: 10.1590/1808-057x201703160

ARAÚJO, Tamires Sousa

Satisfaction among accounting professors in Brazil

v.28, n.74, p.264-281, May/Aug.2017, DOI: 10.1590/1808-057x201703420

ASSUNÇÃO, Renata Rouquayrol

Complexity and corporate governance: an analysis of companies listed on the BM\&FBOVESPA

v.28, n.74, p.213-228, May/Aug.2017, DOI: 10.1590/1808-057x201702660

\section{BAIOCO, Vitor Gomes}

Effects of the audit committee and the fiscal council on earnings quality in Brazil

v.28, n.74, p.229-248, May/Aug.2017, DOI: 10.1590/1808-057x201703250

BALLINI, Rosangela

Value-at-risk modeling and forecasting with range-based volatility models: empirical evidence v.28, n.75, p.361-376, Sep./Dec.2017, DOI: 10.1590/1808-057x201704140

BELTRÃO, Kaizo Iwakami

Executive branch federal civil servant mortality by sex and educational level - 1993/2014

v.28, n.75, p.445-464, Sep./Dec.2017, DOI: 10.1590/1808-057x201704320

BLACK, Roberto

Heterogeneity in earnings quality between different classes of companies after IFRS adoption: evidence from Brazil v.28, n.73, p.113-131, Jan./Apr.2017, DOI: 10.1590/1808-057x201702750 
BORBA, José Alonso

Value Relevance vis-à-vis Disclosure on Business Combinations and Goodwill Recognized by Publicly Traded Brazilian Companies

v.28, n.73, p.77-92, Jan./Apr.2017, DOI: 10.1590/1808-057x201703240

BRAGA, Renata Nogueira

Effects of IFRS adoption on tax avoidance

v.28, n.75, p.407-424, Sep./Dec.2017, DOI: 10.1590/1808-057x201704680

\section{CAMPANI, Carlos Heitor}

Predictive power of Brazilian equity fund performance using $\mathrm{R}^{2}$ as a measure of selectivity v.28, n.74, p.282-296, May/Aug.2017, DOI: 10.1590/1808-057x201703590

\section{CAMPOS, Larissa Couto}

Social quotas, affirmative actions, and dropout in the Business field: empirical analysis in a Brazilian federal university v.28, n.73, p.27-42, Jan./Apr.2017, DOI: 10.1590/1808-057x201702590

\section{CARMONA, Salvador}

Accounting History Research: Scope, Topics and Agenda

v.28, n.75, p.321-325, set./dez.2017, DOI: 10.1590/1808-057x201790210

CARVALHO JÚNIOR, César Valentim de Oliveira

Cognitive brain mapping of auditors and accountants in going concern judgments

v.28, n.73, p.132-147, Jan./Apr.2017, DOI: 10.1590/1808-057x201703430

\section{CHAN, Betty Lilian}

Impacts of the regulatory model for market risk capital: application in a special savings company, an insurance company, and a pension fund

v.28, n.75, p.465-477, Sep./Dec.2017, DOI: 10.1590/1808-057x201703840

COLAUTO, Romualdo Douglas

Voluntary disclosure and earnings management: evidence from the Brazilian capital market

v.28, n.74, p.249-263, May/Aug.2017, DOI: 10.1590/1808-057x201703360

CONSONI, Silvia

Voluntary disclosure and earnings management: evidence from the Brazilian capital market

v.28, n.74, p.249-263, May/Aug.2017, DOI: 10.1590/1808-057x201703360

\section{CORNACCHIONE, Edgard}

Cognitive brain mapping of auditors and accountants in going concern judgments

v.28, n.73, p.132-147, Jan./Apr.2017, DOI: 10.1590/1808-057x201703430

CORREIA, Laíse Ferraz

The effect of asymmetric information risk on returns of stocks traded on the BM\&FBOVESPA v.28, n.75, p.425-444, Sep./Dec.2017, DOI: 10.1590/1808-057x201705230

COSTA, Jose Isidio de Freitas

Using the Newcomb-Benford Law as a Deviation Identification Method in Continuous Auditing Environments: A Proposal for Detecting Deviations over Time v.28, n.73, p.11-26, Jan./Apr.2017, DOI: 10.1590/1808-057x201702690 
COSTA, Patrícia de Souza

Social quotas, affirmative actions, and dropout in the Business field: empirical analysis in a Brazilian federal university v.28, n.73, p.27-42, Jan./Apr.2017, DOI: 10.1590/1808-057x201702590

COSTA, Ruan Rodrigo Araújo da

The relationship between the performance and legal form of microfinance institutions

v.28, n.75, p.377-389, Sep./Dec.2017, DOI: 10.1590/1808-057x201703660

CUNHA, Paulo Roberto da

Influence of board interlocking on earnings management

v.28, n.74, p.179-196, May/Aug.2017, DOI: 10.1590/1808-057x201701980

EGGERT, Neusa Sawczuk von

Why has not it worked? An empirical application of the extended Burns and Scapens' framework within the implementation of a controlling department

v.28, n.73, p.43-60, Jan./Apr.2017, DOI: 10.1590/1808-057x201702820

ESPEJO, Márcia Maria dos Santos Bortolocci

Why has not it worked? An empirical application of the extended Burns and Scapens' framework within the implementation of a controlling department

v.28, n.73, p.43-60, Jan./Apr.2017, DOI: 10.1590/1808-057x201702820

\section{FIGLIOLI, Bruno}

IFRS, synchronicity, and financial crisis: the dynamics of accounting information for the Brazilian capital market v.28, n.75, p.326-343, Sep./Dec.2017, DOI: 10.1590/1808-057x201704450

GALDI, Fernando Caio

Investor relations and information asymmetry

v.28, n.74, p.297-312, May/Aug.2017, DOI: 10.1590/1808-057x201703630

GUZELLA, Marcelo dos Santos

Predictive power of Brazilian equity fund performance using $\mathrm{R}^{2}$ as a measure of selectivity v.28, n.74, p.282-296, May/Aug.2017, DOI: 10.1590/1808-057x201703590

HARTMANN, Frank G. H.

Accounting research: between natural science and practice

v.28, n.73, p.7-10, Jan./Apr.2017, DOI: 10.1590/1808-057x201790160

LAMEIRA, Valdir de Jesus

Predicting financial distress in publicly-traded companies

v.28, n.75, p.390-406, Sep./Dec.2017, DOI: 10.1590/1808-057x201704460

LEMES, Sirlei

IFRS, synchronicity, and financial crisis: the dynamics of accounting information for the Brazilian capital market v.28, n.75, p.326-343, Sep./Dec.2017, DOI: 10.1590/1808-057x201704450

LIMA, Fabiano Guasti

IFRS, synchronicity, and financial crisis: the dynamics of accounting information for the Brazilian capital market v.28, n.75, p.326-343, Sep./Dec.2017, DOI: 10.1590/1808-057x201704450 
LIMA, Gerlando Augusto Sampaio Franco de

Voluntary disclosure and earnings management: evidence from the Brazilian capital market

v.28, n.74, p.249-263, May/Aug.2017, DOI: 10.1590/1808-057x201703360

LONDERO, Paola R.

ICPC 14: what is missing?

v.28, n.75, p.478-485, Sep./Dec.2017, DOI: 10.1590/1808-057x201704740

LUCA, Márcia Martins Mendes De

Complexity and corporate governance: an analysis of companies listed on the BM\&FBOVESPA

v.28, n.74, p.213-228, May/Aug.2017, DOI: 10.1590/1808-057x201702660

MACHADO, Thalyson Renan Bitencourt

Social quotas, affirmative actions, and dropout in the Business field: empirical analysis in a Brazilian federal university v.28, n.73, p.27-42, Jan./Apr.2017, DOI: 10.1590/1808-057x201702590

MACIEL, Leandro dos Santos

Value-at-risk modeling and forecasting with range-based volatility models: empirical evidence

v.28, n.75, p.361-376, Sep./Dec.2017, DOI: 10.1590/1808-057x201704140

MAESTRI, Cláudia Olímpia Neves Mamede

Exposition to Factors of the Investment Funds Market in Brazil

v.28, n.73, p.61-76, Jan./Apr.2017, DOI: 10.1590/1808-057x201702940

MAJOR, Maria João

Positivism and "alternative" accounting research

v.28, n.74, p.173-178, maio/ago.2017, DOI: 10.1590/1808-057x201790190

MALAQUIAS, Rodrigo Fernandes

Exposition to Factors of the Investment Funds Market in Brazil

v.28, n.73, p.61-76, Jan./Apr.2017, DOI: 10.1590/1808-057x201702940

MARQUES, Felipe Tumenas

Impacts of the regulatory model for market risk capital: application in a special savings company, an insurance company, and a pension fund

v.28, n.75, p.465-477, Sep./Dec.2017, DOI: 10.1590/1808-057x201703840

MARTINS, Eliseu

Challenges with the public policy of measuring assets to set tariffs in the electricity sector: should someone benefit and someone be sacrificed?

v.28, n.75, p.344-360, Sep./Dec.2017, DOI: 10.1590/1808-057x201703160

MEDINA, Fabio Augusto Scalet

Geographically Weighted Logistic Regression Applied to Credit Scoring Models

v.28, n.73, p.93-112, Jan./Apr.2017, DOI: 10.1590/1808-057x201703760

MIRANDA, Gilberto José

Satisfaction among accounting professors in Brazil

v.28, n.74, p.264-281, May/Aug.2017, DOI: 10.1590/1808-057x201703420 
MIRANDA, Gilberto José

Social quotas, affirmative actions, and dropout in the Business field: empirical analysis in a Brazilian federal university v.28, n.73, p.27-42, Jan./Apr.2017, DOI: 10.1590/1808-057x201702590

MONTEZANO, Roberto Marcos da Silva

Predicting financial distress in publicly-traded companies

v.28, n.75, p.390-406, Sep./Dec.2017, DOI: 10.1590/1808-057x201704460

NAKAO, Sílvio Hiroshi

Heterogeneity in earnings quality between different classes of companies after IFRS adoption: evidence from Brazil v.28, n.73, p.113-131, Jan./Apr.2017, DOI: 10.1590/1808-057x201702750

NAKAO, Sílvio Hiroshi

Unconditional conservatism in Brazilian public companies and tax neutrality

v.28, n.74, p.197-212, May/Aug.2017, DOI: 10.1590/1808-057x201702450

OLIVEIRA, Fernando Nascimento de

Predicting financial distress in publicly-traded companies

v.28, n.75, p.390-406, Sep./Dec.2017, DOI: 10.1590/1808-057x201704460

PEREIRA, Janser Moura

Satisfaction among accounting professors in Brazil

v.28, n.74, p.264-281, May/Aug.2017, DOI: 10.1590/1808-057x201703420

PICCOLI, Marcio Roberto

Influence of board interlocking on earnings management

v.28, n.74, p.179-196, May/Aug.2017, DOI: 10.1590/1808-057x201701980

REZENDE, Felipe Fontaine

Predicting financial distress in publicly-traded companies

v.28, n.75, p.390-406, Sep./Dec.2017, DOI: 10.1590/1808-057x201704460

ROCHA, Armando Freitas da

Cognitive brain mapping of auditors and accountants in going concern judgments

v.28, n.73, p.132-147, Jan./Apr.2017, DOI: 10.1590/1808-057x201703430

ROCHA, Fábio Theoto

Cognitive brain mapping of auditors and accountants in going concern judgments

v.28, n.73, p.132-147, Jan./Apr.2017, DOI: 10.1590/1808-057x201703430

RODRIGUES, Sandrielem da Silva

Investor relations and information asymmetry

v.28, n.74, p.297-312, May/Aug.2017, DOI: 10.1590/1808-057x201703630

SANTOS, Ariovaldo dos

ICPC 14: what is missing?

v.28, n.75, p.478-485, Sep./Dec.2017, DOI: 10.1590/1808-057x201704740

SILVA, Alan Ricardo da

Geographically Weighted Logistic Regression Applied to Credit Scoring Models

v.28, n.73, p.93-112, Jan./Apr.2017, DOI: 10.1590/1808-057x201703760 
SILVA, Wilton Bernardino da

Using the Newcomb-Benford Law as a Deviation Identification Method in Continuous Auditing Environments: A Proposal for Detecting Deviations over Time

v.28, n.73, p.11-26, Jan./Apr.2017, DOI: 10.1590/1808-057x201702690

SIQUEIRA, Leonardo Souza

The effect of asymmetric information risk on returns of stocks traded on the BM\&FBOVESPA

v.28, n.75, p.425-444, Sep./Dec.2017, DOI: 10.1590/1808-057x201705230

SOUZA, Maíra Melo de

Value Relevance vis-à-vis Disclosure on Business Combinations and Goodwill Recognized by Publicly Traded Brazilian Companies

v.28, n.73, p.77-92, Jan./Apr.2017, DOI: 10.1590/1808-057x201703240

SUGAHARA, Sonoe

Executive branch federal civil servant mortality by sex and educational level - 1993/2014

v.28, n.75, p.445-464, Sep./Dec.2017, DOI: 10.1590/1808-057x201704320

TRAVASSOS, Silvana Karina de Melo

Using the Newcomb-Benford Law as a Deviation Identification Method in Continuous Auditing Environments: A Proposal for Detecting Deviations over Time

v.28, n.73, p.11-26, Jan./Apr.2017, DOI: 10.1590/1808-057x201702690

VALE, Juliana Pinhata Sanches do

Unconditional conservatism in Brazilian public companies and tax neutrality

v.28, n.74, p.197-212, May/Aug.2017, DOI: 10.1590/1808-057x201702450

VASCONCELOS, Alessandra Carvalho de

Complexity and corporate governance: an analysis of companies listed on the BM\&FBOVESPA

v.28, n.74, p.213-228, May/Aug.2017, DOI: 10.1590/1808-057x201702660 